\title{
Characterizing and Modeling the Precursors to Coarse Grain Formation during Beta-Annealing of Ti-6Al-4V
}

\author{
A.L. Pilchak ${ }^{1}$, S. Srivatsa ${ }^{2}$, N.C. Levkulich ${ }^{3}$, \\ V. Sinha ${ }^{3}$, E.J. Payton ${ }^{1}$, and S.L. Semiatin ${ }^{1}$ \\ ${ }^{1}$ Air Force Research Laboratory, AFRL/RXCM, Wright Patterson AFB OH \\ ${ }^{2}$ Srivatsa Consulting, LLC \\ ${ }^{3}$ UES Inc., Beavercreek, OH
}

\begin{abstract}
$\underline{\text { Abstract }}$
Coarse prior $\beta$ grains exceeding $3 \mathrm{~mm}$ in diameter have been sporadically observed following $\beta$ annealing of $\alpha+\beta$ forged titanium alloys. Recent work has shown that the occurrence of coarse grains may be due in part to the stabilization of a $\{001\}<110>$ texture during hot working that was further enhanced in intensity at the expense of other texture components during the early stages of $\beta$ annealing. With the majority of the material comprised of low misorientation subgrains of a single texture component, the nuclei for coarse grains was the minority fraction of grains that were highly misoriented, and therefore had boundaries with higher energy and mobility, compared to the average grain. In this work, Ti-6Al-4V bar was side-pressed to various reductions in the $\alpha+\beta$ phase field to further investigate the role of texture and the effects of strain, strain-path, and deformation heating on the propensity to form abnormally large grains during $\beta$-annealing. The experiments were interpreted in the context of a continuum finite element model and viscoplastic self-consistent crystal plasticity simulations. Based on the results from experiment and modeling, we make recommendations with respect to the $\alpha+\beta$ forging process to avoid the occurrence of excessively coarse $\beta$ grains.
\end{abstract}

\section{Introduction}

A wide range of property combinations can be obtained in titanium alloys via judicious choice of thermomechanical processing (TMP) parameters. Depending on overall design methodology, $\alpha / \beta$ titanium parts for rotating applications are typically fabricated by hot working and heat treatment in the $\alpha /$ $\beta$ field. By contrast, structural components for airframes are most often $\alpha / \beta$ worked followed by supertransus ( $\beta$ ) annealing. Such TMP sequences lead to prior $\beta$ grains whose size is relatively large $(\sim 500$ to $2500 \mu \mathrm{m})$ and which contain a transformation product of colony or Widmanstatten $\alpha$ whose fineness is dependent on local cooling rate. These microstructures provide substantial benefits with regard to fatigue crack growth resistance and fracture toughness at the expense of strength, ductility, and high-cycle-fatigue resistance [2-4].

Because of the importance of prior $\beta$ grain size with respect to the balance of properties in $\beta$-annealed titanium components, a large amount of research has been devoted to understanding and modeling it [6-12]. These works show that crystallographic texture is a key contributor to the experimentally observed variability in grain growth kinetics. In fact, texture effects were shown to have a first-order effect on grain growth and could lead to graingrowth exponents in the range of 2 to 6 , sequential periods of slow and rapid growth. To quantify the so-called texture-controlled grain-growth behavior, Monte-Carlo (MC) modeling methods were developed and were successful in providing at least qualitative descriptions of the prior observations [13-14]. MC approaches have also been developed and applied to predict the occurrence of abnormal grain growth (AGG) during the annealing of single-phase metallic materials [e.g., 15]. By this means, the importance of the intensity and breadth of a minor texture component on AGG have been demonstrated. AGG has also been observed in two-phase $(\alpha / \beta)$ titanium alloys such as Ti-6Al-4V subjected to subtransus plane-strain hot working (e.g., plate rolling) followed by $\beta$ annealing [16]. The occurrence of AGG in these cases has been attributed to macroscopic variations in deformation texture across the workpiece section. More recently, AGG has been observed during the $\beta$ annealing of forgings whose prior (subtransus) deformation was also largely plane strain in nature. Per the findings of Pilchak, et al. [17], the formation of grain-size heterogeneity in such cases may arise from the development of texture heterogeneity on a relatively local scale prior to and during early times in the $\beta$-annealing process. As in the work of Ivasishin and coworkers [1115], the growth of a small volume fraction of grains associated with a minor texture component into those of a major texture component may be the 
source of AGG in these cases also.

The objective of the present research was to elucidate experimentally the mechanisms of the formation of coarse-grain regions in Ti-6Al-4V forgings and interpret such occurrences in light of the evolution of non-uniform textures. We use the term "coarse grain" (CG) to describe this phenomenon as the mechanism of formation is different than classical AGG mechanisms. To meet these goals, a series of Ti-6Al-4V bars were conventionally sidepressed to produce a nominally plane-strain mode of deformation. Texture and grain structure development during subsequent $\beta$ annealing were determined via electron-backscatter diffraction (EBSD) and interpreted using continuum level FEM simulations of the macroscopic strain state, mesoscale (viscoplastic, self-consistent) simulations of the accompanying deformation-texture evolution, and the concept of oriented growth during the early stages of annealing.

\section{Materials and experiments}

In this work, Ti-6.29Al-3.8V-0.22Fe-0.17O bars each measuring $63.5 \mathrm{~mm} \varnothing \mathrm{x} 190.5 \mathrm{~mm}$ long were heated to $954 \mathrm{C}$, held for $90 \mathrm{minutes}$, and then forged on $38 \mathrm{C}$ dies in a side-pressed configuration with a ram speed of $25.4 \mathrm{~mm} / \mathrm{s}$ to total reductions of $42.6 \%, 64.5 \%$, and $69 \%$. The forgings were cross sectioned at the mid-span perpendicular to the bar axis and also sectioned longitudinally at the center of the forging in order to provide coupons for quantitative texture analysis via electron backscatter diffraction (EBSD) and also for $\beta$ annealing. The latter samples were heat treated by inserting into a furnace at $538 \mathrm{C}$ after which the furnace temperature was increased $149 \mathrm{C}$ per hour to $1038 \mathrm{C}$ (to simulate the slow heating rates encountered in large structural forgings), soaking for $1 \mathrm{hr}$, and then air cooling. The samples were prepared with standard procedures involving grinding with successively finer SiC papers (to 800 grit), intermediate polishing with diamond paste, and final polishing with colloidal silica. EBSD was performed in an FEI XL30 at an accelerating voltage of $20 \mathrm{kV}$ at spot size $5 \mathrm{using}$ a $100 \mu \mathrm{m}$ final aperture resulting in $\sim 22 \mathrm{nA}$ current. Multi-tile scans were collected at step sizes of $0.2 \mu \mathrm{m}$ and $5 \mu \mathrm{m}$ in order to investigate the $\alpha$ and $\beta$ phase textures. The EBSD data were analyzed using TSL OIM Analysis and MTEX [18].

A 3D finite element model (FEM) of the forging process, including transfer and resting, was developed in DEFORM ${ }^{\mathrm{TM}}$. The heat transfer (h) and friction ( $\mathrm{m}$ ) coefficients were iteratively tuned until the model predicted the correct deformed shape and load-stroke behavior, finally arriving at $\mathrm{m}=0.25$ and $\mathrm{h}=$ $0.0020 \mathrm{BTU} / \mathrm{sec} / \mathrm{in} 2 / \mathrm{F}$ (h during the $3 \mathrm{~s}$ rest was $1 / 3$ of this). The TMP histories of nine locations in the forging were extracted from the FEM, which had 100,000 elements. Interpretation of the deformation textures in relation to the location-specific strain-paths were aided by viscoplastic self-consistent (VPSC) crystal plasticity [19]. All simulations were performed with VPSC7d using the neff $=10$ approximation which represents a middle ground between the stiff isostrain-rate and compliant isostress approximations and assuming no hardening. The ratios of slip system strengths mirrored Dunst and Mecking [20], $\left\{\alpha \_\right.$prism, $\left.\alpha \_b a s a l, \alpha \_\left(p y r \_a\right), \alpha \_\left(p y r \_c+a\right)\right\}=\{1,1.5,1,3\}$ while pencil glide on $\{110\},\{112\}$, and $\{123\}$ was assumed in the $\beta$ phase at a ratio of 0.33 to prism slip. VPSC simulations were used to explore the effect of strain path and volume fraction $\alpha p$ on texture evolution. To this end, simulations were performed to a true strain of 1.2 using steps of 0.025 imposing plane strain compression (PSC), axisymmetric compression, and strainpaths intermediate to these. It is also noteworthy that the VPSC formulation considers grain morphology evolution and relaxed constraints are introduced naturally as the beta grain shape becomes pancaked.

\section{Results and discussion}

The primary results from this research included observations of the as-forged and $\beta$-annealed microstructures and textures, assessment of local thermomechanical histories in locations that did and did not exhibit CG formation, and interpretation of these results using the self-consistent crystal plasticity formulation.

The as forged bars and the corresponding deformed shapes predicted by the FE model are shown in Figure 1. At the midspan, the reduction in thickness was accommodated entirely by extension in the PMFD. In contrast, lateral flow was restricted with increasing distance from the centerline (e.g. Figure 1a), which necessitated flow in the axial direction as well. This is also evident from the barreling at the ends of the original bar (Figure 1b). Polarized light optical micrographs of mid-span cross-section of the as-forged and forged + heat treated microstructures of bars side pressed to $64.5 \%$ and $69 \%$ are shown in Figure 2, which also contains the final locations of five tracked. No strong flow lines were evident in the as-forged condition, though subtle variations in contrast were evident through thickness and they were symmetric about the midplane of the forging. The dead metal regions were noticeably lighter than the center of the forging, which appeared much darker. A moderately sized region at the center of the $64.5 \%$ and $69 \%$ forging formed prior $\beta$ grains $\sim 3-5 \mathrm{x}$ larger than the nominal microstructure. By contrast, no such gradients were observed in the forging reduced by $42.6 \%$ and no CGs were 
observed upon $\beta$ annealing.
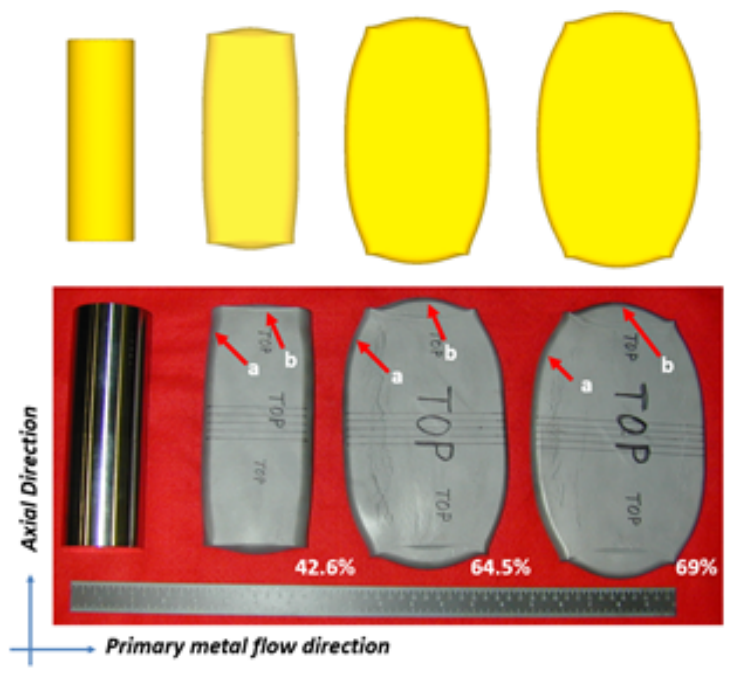

Figure 1: As-forged bars pressed to various total reductions and the corresponding deformed shape of the FE model. Note the decrease in lateral flow at location (a) which necessitates a progressive increase in barreling at location (b).
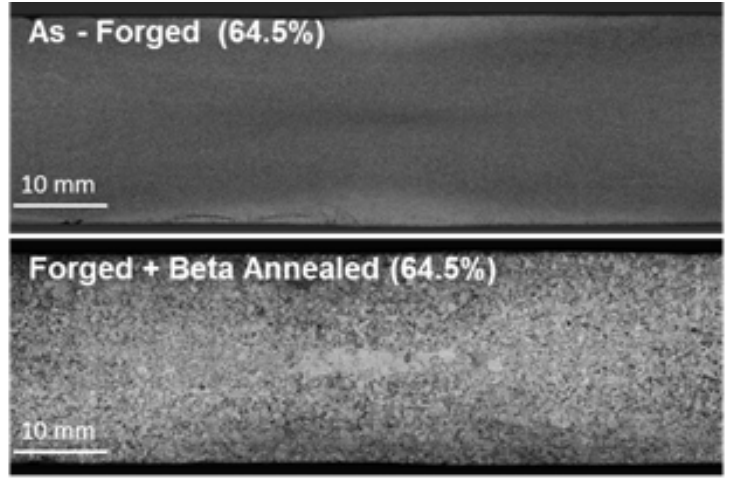

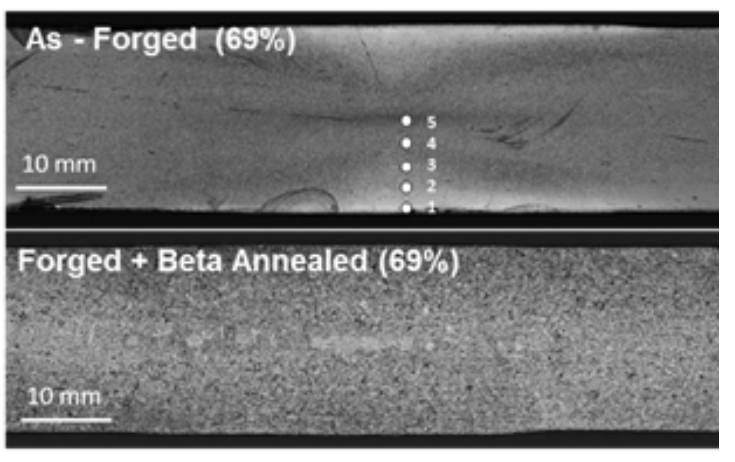

Figure 2: Polarized light optical micrographs of the as-forged and forged + beta-annealed conditions for reductions of $64.5 \%$ and $69 \%$. Points $1-5$ correspond to regions that were point tracked in the FE model.

The $\alpha$ and $\beta$ phase textures at the center of the $69 \%$ as-forged condition are shown in Figure 3. Due to the allotropic transformation on cooling, the $\alpha$ phase texture is a mixture of deformation and transformation texture components with the latter arising from $\alpha$ orientations related to the $\beta$ phase deformation texture through the Burgers orientation relation, viz. (0001) $\|\{110\}$ and $\langle 11 \overline{2} 0>\|<111>$, hence the correspondence between the $\beta 110$ and $\alpha$ 0001 pole figures. The principal $\alpha$ and $\beta$ phase deformation texture components were consistent with orientations known to form during PSC of single 
phase HCP and BCC metals $[20,21]$, which is consistent with the strain-path predicted by the FEM in this location $\left(\varepsilon_{-} \mathrm{PMFD}=2.1, \varepsilon_{-}\right.$axial $=0.1$ $\varepsilon_{-}$forging $=-2.2$ ). The $\alpha$ phase texture consisted largely of 0001 poles parallel to the axial direction of the billet and additional intensities in locations of high intensity in the $\beta$ phase 110 pole figure. The $\beta$ phase deformation texture consisted primarily of orientations clustered near the ends of the $<110>\|$ PMFD partial fiber (also referred to as the $\alpha$-fiber of typical BCC PSC or rolling textures [21]). Specifically, these were the rotated cube $\{001\}<110>$ and $\{110\}<011>$ components $(\sim 18 \mathrm{x}$ and $\sim 8 \mathrm{x}$ random in ODF, respectively, and expressed in terms of \{forging plane $\})$ and there was a notable absence of orientations at $\{111\}<110>$. It is noteworthy that the rotated cube orientation has been previously identified as a contributor to accelerated grain growth during the early stages of $\beta$ annealing owing to its existence as a network of mostly low angle, low energy, and low mobility boundaries with a small number of highly misoriented, high energy, and high mobility boundaries. These high mobility boundaries migrate rapidly to consume the stored work associated with the low angle dislocation walls separating the subgrain boundaries [17].
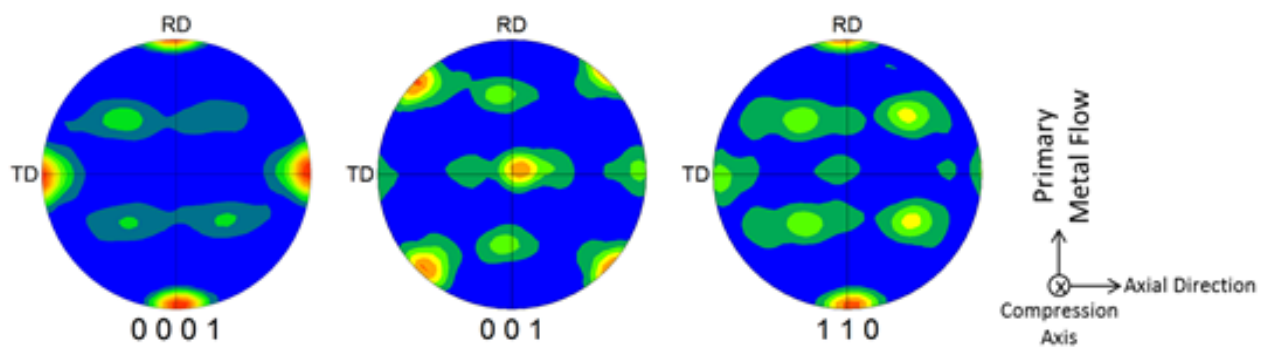

Figure 3: Directly measured alpha and beta phase pole figures for the as-forged condition (69\% reduction). Contours $1,2,3 \ldots . . m a x$. Refeer to the reference frame on the right hand side of the figure.

One half of the remaining cross-sectioned forging was beta annealed and cut parallel to the original bar axis in order to assess the extent of CG formation in the plane perpendicular to the primary metal flow direction (PMFD). As shown in Figure 4, CGs were observed at the midplane of the forging from the mid-span up to approximately $42 \mathrm{~mm}$ from the edge of the barreled region at the top of the forging as shown in Figure 1. A representative EBSD scan and the associated recalculated $\beta$ phase orientations at location 8 are shown in Figure 5 . This figure shows the $\beta$ grains in the center of the forging are at least $3 \mathrm{x}$ larger than the adjacent grains in the forging direction, and generally have low angle boundaries between them resulting in a large single-crystal-like $\beta$ grain in the axial and primary metal flow directions.

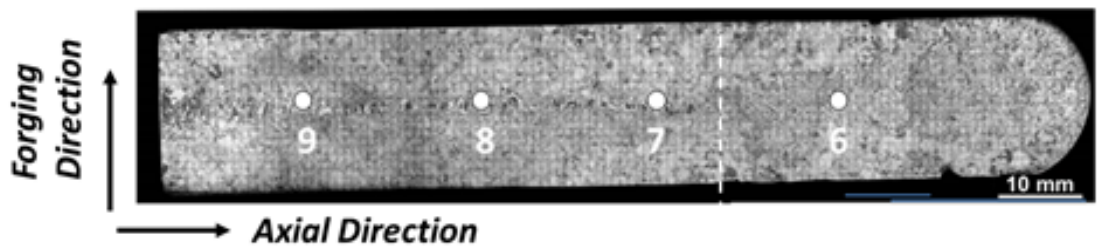

Figure 4: Polarized light optical micrograph of the forged + beta-annealed condition perpendicular the primary metal flow direction. Points 6-9 correspond to regions that were point tracked in the FE model and the vertical white line corresponds to the extent of coarse grain formation. 

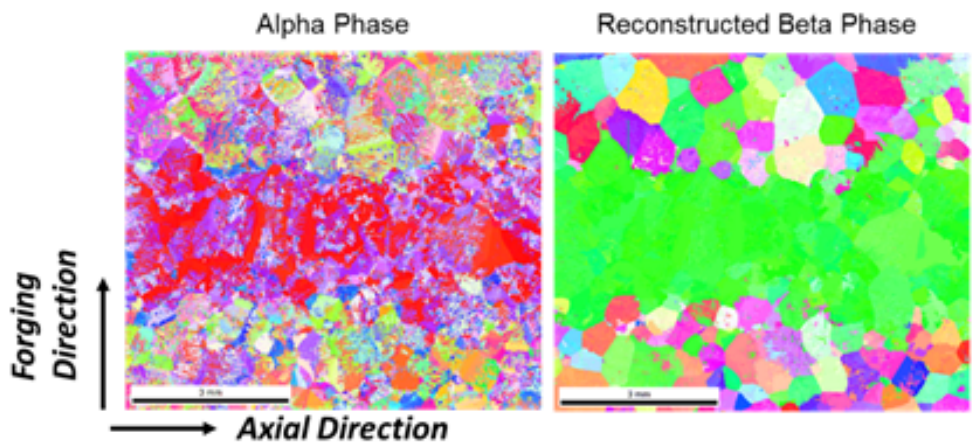

Figure 5: Primary metal flow direction inverse pole figure maps showing the measured alpha and reconstructed beta phase orientations corresponding to location 8 in the forging.

The thermomechanical histories corresponding to five locations in and four locations outside of the CG region are compared in Figure 6a which show the three principal strain components over the deformation history and also whether or not a particular location exhibited CGs upon $\beta$ annealing. As expected, strains were generally highest in the forging direction and these were largely balanced by nearly equal strain in the PMFD. Looking at the forging and PMFD strains, we see essentially two classes - points 1-3 are in regions of the forgings that cool relatively quickly and hence increase in flow stress and do not accumulate much strain at all. Points 4-9 experience much higher forging direction strains due to their position relative to the midplane of the forging. Among these, point 6 is a unique outlier. It is the only location that achieved relatively high strain in both the primary metal flow and forging directions yet did not exhibit $\mathrm{CG}$ growth following $\beta$ annealing. Recall that the material is incompressible and hence $\varepsilon_{-}{ }_{-} \mathrm{PMFD}+\varepsilon^{\prime}$ axial $+\varepsilon$ _ forging $=0$. Hence, any difference in strain between the PMFD and forging direction must be accommodated by flow in the axial direction (along the original bar axis) and this is the distinguishing feature of location 6 . As illustrated later, these changes in strain-path alter the development of the rotated cube deformation texture component, which is a necessary precursor for CG growth. The formation of such a texture is driven by the degree of PSC deformation imposed at a particular location and hence it is convenient to look at the ratio $\varepsilon_{\text {forging }} / \varepsilon_{P M F D}$ (Figure $6 \mathrm{~b}$ ). Perfect plane strain compression occurs when $\varepsilon_{\text {forging }} / \varepsilon_{P M F D}=-1$ and there is no axial extension. In contrast, under axisymmetric compression metal flow is equal in all directions perpendicular to the principle forging direction with magnitude equal to half the forging direction strain, $\varepsilon_{f o r g i n g} / \varepsilon_{P M} F D=-1 / 0.5=-2$. Hence, from Figure $6 \mathrm{~b}$ it is clear that 6 of 9 data points are subjected to PSC. All of these exhibited CG formation except for points 2 and 3 , which experienced peak axial strains of $\sim 0.48$ and 0.97 , respectively. Point 1 is situated near the die/workpiece interface and hence experiences significant chilling during transfer and resting which resulted in a locally elevated flow stress and very minimal strain accumulation $\left(\varepsilon \_\right.$forging $\left.=0.05\right)$. Axial strain initially accommodates $\sim 15 \%$ of the deformation at points 6 and 7 and this fraction increases to $\sim 51 \%$ and $\sim 23 \%$, respectively, with increasing reduction. There was a notable absence and presence of CGs at these locations, respectively. The arrows in Figure 6a highlight the strain history for point 6 which

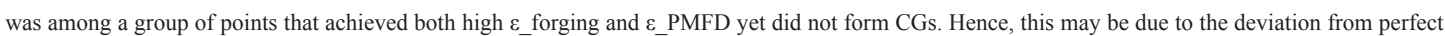
PSC that is afforded by the higher $\varepsilon_{-}$axial at this location compared to the others. For example, the $\beta$ phase deformation texture at this location (Figure 7) reveals the presence of an 001 partial fiber aligned with the forging direction with some minor preferential alignment of $<110>$ with the PMFD. Consistent with the strain path at this location, such a texture can be described as a mixture of an axisymmetric compression and PSC deformation textures with the 001 partial fiber and the alignment of $<110>$ corresponding to the former and latter, respectively. In contrast, we also observed a moderately strong rotated cube texture at location 5 which formed CGs upon $\beta$-annealing. These results further confirm the identification of the BCC rotated cube texture as a precursor to $\mathrm{CG}$ formation [17]. 

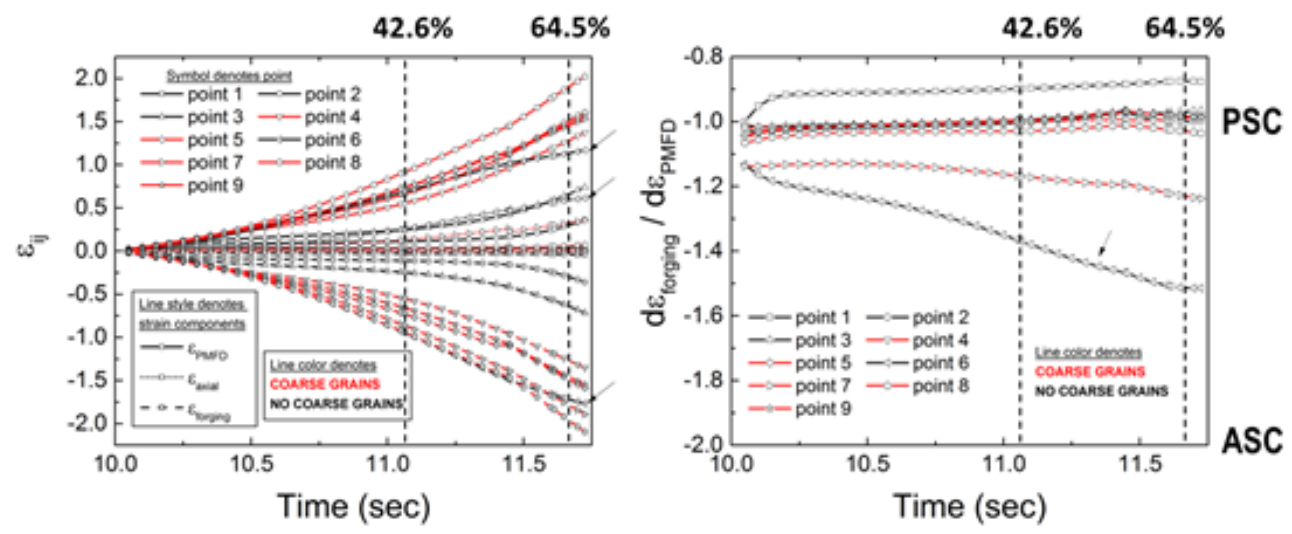

Figure 6: (a) Thermomechanical histories and (b) instantaneous ratio of forging-to-PMFD strain corresponding to points 1-9 in the forging . Vertical lines in (a) and (b) correspond to intermediate reductions and strain ratios corresponding to PSC and axisymmetric compression (ASC) are shown for reference in (b). No coarse grains were observed at any location in the forging reduced to $42.6 \%$.
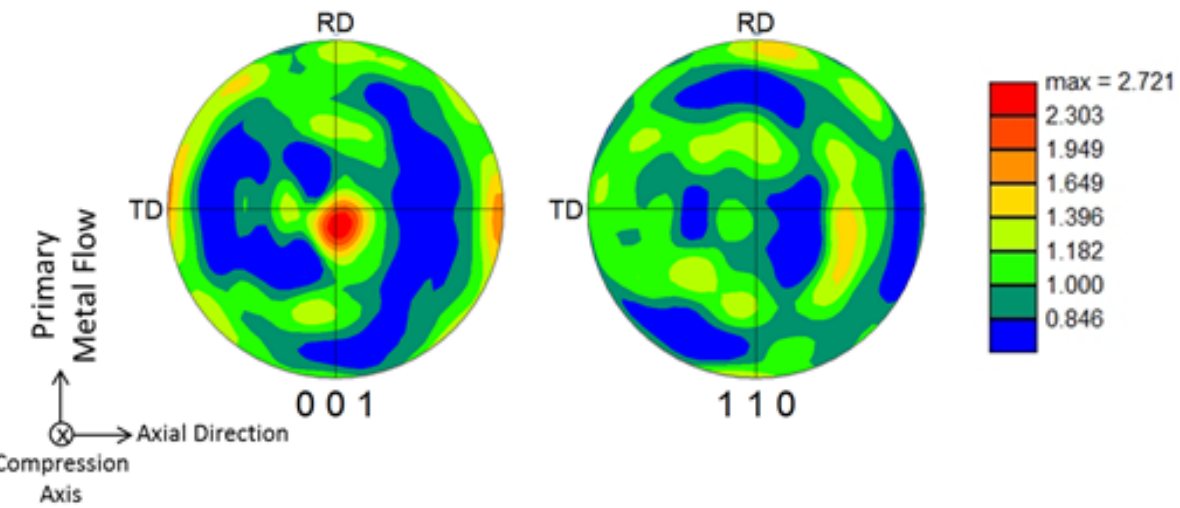

Figure 7: Directly measured beta phase deformation texture at location 6 highlighting the predominance of the 001 partial-fiber over the rotated cube. Refeer to the reference frame on the left hand side of the figure.

We hypothesize that the change in local constraint and the additional ability to flow axially precludes the formation of the rotated cube texture, which effectively shuts down CG formation. This hypothesis was investigated with a series of VPSC simulations (Vf $\alpha p=25 \%)$ where we ease the plane strain boundary condition and allow increasing fractions of $\varepsilon_{-}$axial at the expense of $\varepsilon_{-}$PMFD. The results (Figure 8 ) are shown on $\varphi 2=450$ sections of the orientation distribution function (ODF), which show the key features of PSC and axisymmetric deformation textures. There are a few key features of the deformation texture and its evolution which can be interpreted in the context of fibers and specific orientations $\{\mathrm{hkl}\}$ that lie on them (Figure 9). 

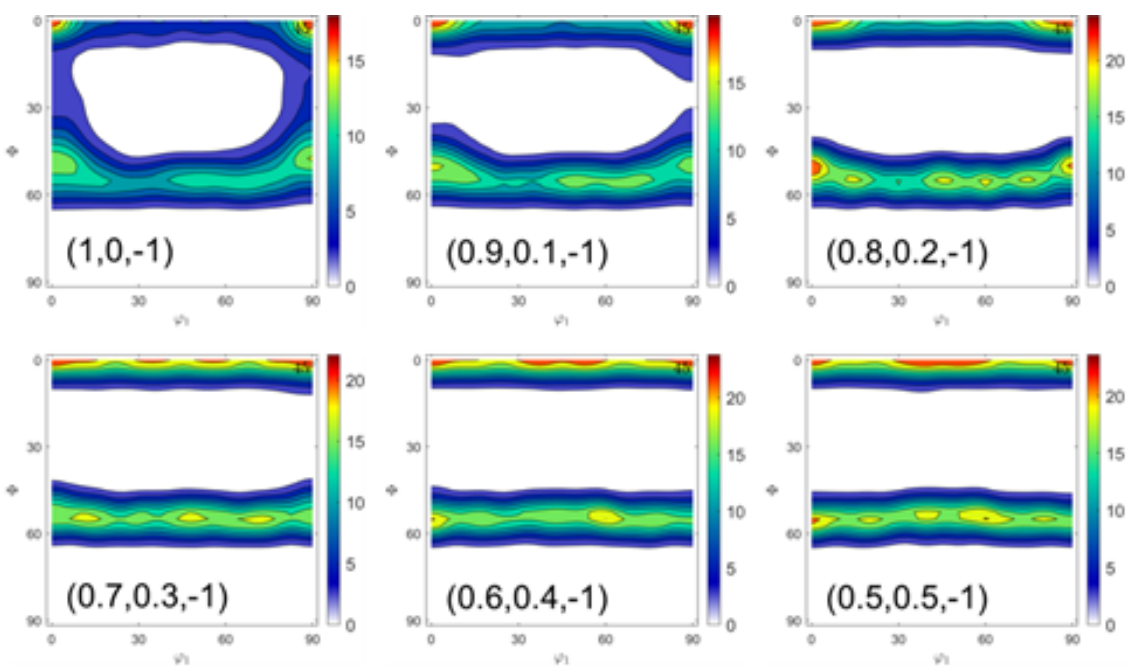

Figure 8: Effect of strain-path on the evolution of an initially random texture for an alpha vol. frac. of $25 \%$. Velocity gradient (strain-rate) components in the form $\left(L \_11,22, L \_33\right)$ are shown and represent a transition from perfect plane strain compress $(1,0,-1)$ to perfect axisymmetric compression $(0.5,0.5,-1)$. No shear strains were considered. All texures are represented on $\phi_{2}=45^{\circ}$ sections.

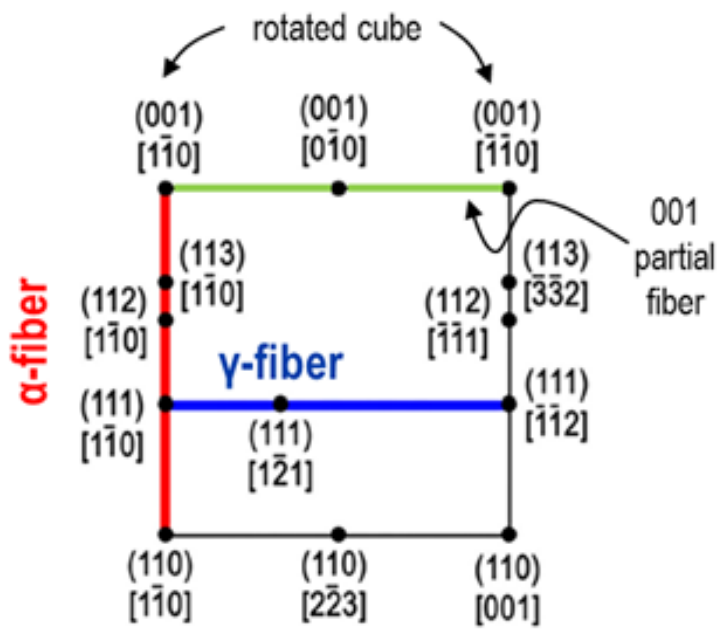

Figure 9: Ideal BCC Plane Strain Compression Deformation Texture Components on the $\varphi 2=450$ section of the ODF. \{hkl\} correspond to crystallographic planes and directions parallel to the rolling plane and rolling direction, respectively.

Under perfect PSC, we observe the formation of a weak, $<5 \mathrm{x}$ random, 001 partial fiber that terminates into strong rotated cube orientations ( 18x). In 
addition, we see moderate intensities along the $\gamma$-fiber $(10 \mathrm{x})$ and a pathway where orientations are flowing from the $\gamma$-fiber along the $\alpha$-fiber up to the rotated cube orientation. As the PSC boundary conditions are eased and 10\% of the strain is accommodated axially, we first see the pathway between the $\alpha$ - and $\gamma$-fibers is eliminated and hence we observe a slight increase in the density of orientations on the $\gamma$-fiber. There is also a slight increase in the intensities present along the 001 partial fiber. With $20 \%$ axial deformation, we see a continued redistribution of orientations along the 001 partial fiber though there is still slight a preference for the rotated cube orientation and a notable intensity at $\{111\}<110>$. This preference diminishes and is eliminated by $30 \%$ axial deformation where we see a relatively homogeneous distribution of orientations along the $\gamma$-fiber. Axisymmetric compression, which occurs when the forging direction strain is equally accommodated by the principal strains perpendicular to it, produces a texture that is essentially equivalent to that at $40 \%$ axial deformation. Both show well developed $\gamma$ - and 001 partial fibers with high intensities (18 23x) present along the entire fiber. It is worth mentioning that the VPSC simulations predict a $\gamma$-fiber which is notably absent from the experimental deformation texture in the $69 \%$ as-forged condition. Simulations which used boundary conditions extracted from streamlines in the FEM, including rigid body rotation due to metal flow and minor off-axis shears, do not predict the formation of the $\gamma$-fiber implying that it is relatively unstable compared to the $\alpha$-fiber and the rotated cube orientation. These simulations will be the subject of a future manuscript.

The deformation texture, however, does not provide a complete description of the texture present as the $\beta$ transus temperature is exceeded during $\beta$ annealing, all primary $\alpha$ phase is dissolved and the $\beta$ grain boundaries are unpinned. Because there are a wide range of orientations present in the deformation texture, it is plausible that all of these orientations serve as sites for epitaxial growth of $\beta$ phase as the $\alpha$ phase dissolves and hence we would expect a variety of high angle grain boundaries to form as the $\beta$ transus temperature is exceeded and normal, curvature-controlled grain growth to occur. This is not observed experimentally, however, as shown by recent short-duration annealing experiments on Ti-6Al-4V sheet [17]. These results show that there is a dramatic increase in the volume fraction of rotated cube component during the early stages of annealing. So much so, in fact, that it overtakes essentially all other texture components. The exact mechanism remains the subject of ongoing investigation, but it may be related to the phenomenon of orientation-dependent stored work that has been observed in a number of BCC transition metals [22]. As a highly symmetric orientation, the rotated cube requires only four active $\{110\}<111>$ slip systems to stabilize it with respect to PSC and hence does not develop strong orientation gradients. In addition to having a low Taylor factor, the dislocations that are present have very limited elastic interactions resulting in a very limited driving force for recrystallization. All of these aspects may give the rotated cube orientation an advantage to grow and consume other $\beta$ phase orientations that may be encountered during the early stages of annealing.

Considering the experimental and computational results, it may be possible to eliminate CG formation by avoiding PSC, if possible, or by controlling the amount of strain in a given step if it must be imposed in PSC. Specifically, the undesirable rotated cube texture can be avoided if one designs a forging process that forces at least $\sim 25 \%$ axial extension. If, on the other hand, PSC cannot be avoided due to other constraints, the true strain imposed in the forging direction should not exceed 1.0. As a final strategy, Morris [23] showed that lower forging temperatures, which can be achieved through lower preheat temperature or by using slower strain rates in cases where there is significant deformation heating, may reduce the propensity for CG formation. Mechanistically, more strain will partition to the $\alpha$ p phase and hence slow the evolution of the $\beta$ phase texture. Indeed, VPSC simulations predict that the rotated cube is destabilized with increasing volume fraction of $\alpha$ p (Figure 10). 

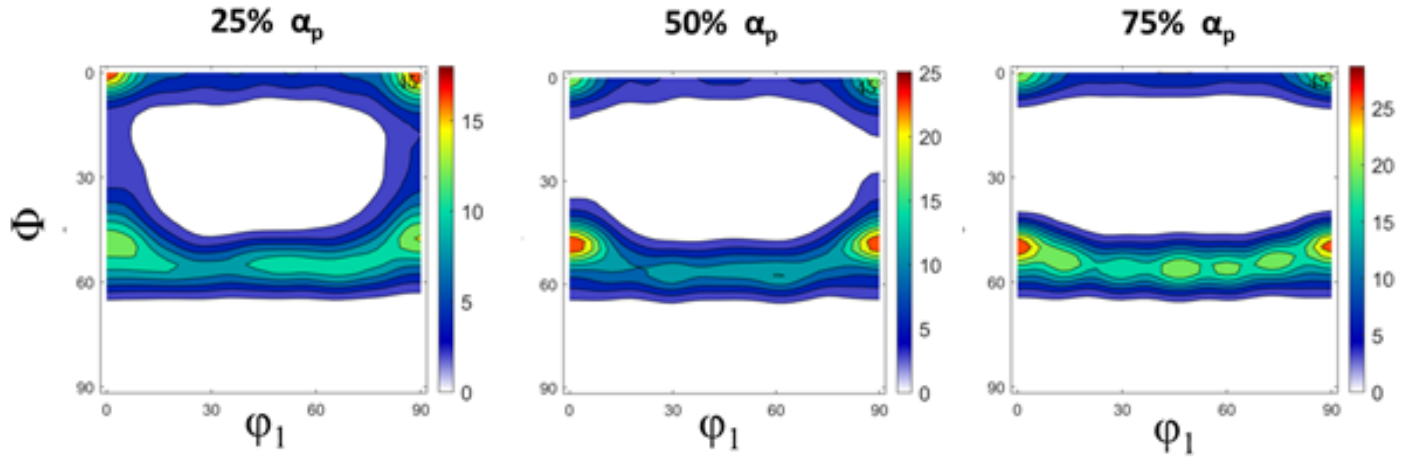

Figure 10:Effect of primary alpha volume fraction on beta phase texture evolution after a true strain of 1.2 in PSC from an initially random texture. All texures are represented on $\phi_{2}=45^{\circ}$ sections.

\section{$\underline{\text { Summary and conclusions }}$}

In summary, $\alpha+\beta$ side pressing Ti- $6 \mathrm{Al}-4 \mathrm{~V}$ bar followed by $\beta$-annealing led to the formation of coarse $\beta$ grains in regions of the forging that experienced primarily PSC deformation. The FEM indicated that locations with CGs had strain paths that closely approximated plane strain compression and locations which deviated from PSC did not exhibit CGs. The PSC strain path was found experimentally and via crystal plasticity to promote the rotated cube $\{001\}<110>$ deformation texture, which confirmed a previously reported link between this orientation and the occurrence of CG formation upon $\beta$ annealing. VPSC simulations revealed that the rotated cube orientation the was strongest under PSC deformation and became increasingly less prevalent as the PSC boundary conditions were eased toward axisymmetric deformation paths which afforded flow along the original bar axis. Considering the experimental and computational results, the best strategy to avoid CGs in $\beta$-annealed forgings is to avoid imposing strains $>1.0$ in PSC and striving for an intermediate strain-path where at least $\sim 25 \%$ of the forging direction strain is accommodated by flow in the axial direction.

\section{$\underline{\text { References }}$}

1. G. Luetjering and M. Peters: Report CS-2933, Electric Power Research Institute, Palo Alto, CA, 1983.

2. G. Luetjering: Mater. Sci. Eng. A, 1998, vol. A243, pp. 32-45.

3. G. Luetjering and J.C. Williams: Titanium, Springer Verlag, Berlin, 2007.

4. S.L. Semiatin, V. Seetharaman, and I. Weiss: Advances in the Science and Technology of Titanium Alloy Processing, I. Weiss, et al., eds., TMS, Warrendale, PA, 1997, p. 3.

5. S.L. Semiatin, S.L. Knisley, P.N. Fagin, F. Zhang, and D.R. Barker: Metall. and Mater. Trans. A, 2003, vol. 34A, pp. $2377-2386$.

6. S.L. Semiatin, J.C. Soper, and I.M. Sukonnik: Scripta Metall. Mater., 1994, vol. 30, pp. 951-955.

7. S.L. Semiatin, J.C. Soper, and I.M. Sukonnik: Acta Mater., 1996, vol. 44, pp. 1979-1986.

8. S.P. Fox, in: F.H. Froes and I.L. Caplan. (Eds.), Titanium'92, Science and Technology, TMS, Warrendale, PA, 1992, pp. 769-776.

9. S.L. Semiatin, P.N. Fagin, M.G. Glavicic, I.M. Sukonnik, and O.M. Ivasishin: Mater. Sci. Eng. A, 2001, vol. A299, pp. 225-234.

10. F.G. Gil and J.A. Planell: Mater. Sci. Eng. A, 2000, vol. A283, pp. 17-24.

11. O.M. Ivasishin, S.V. Shevchenko, and S.L. Semiatin: Mater. Sci. Eng. A, 2002, vol. A332, pp. 343-350.

12. O.M. Ivasishin, S.L. Semiatin, P.E. Markovsky, S.V. Shevchenko, and S.V. Ulshin: Mater. Sci. Eng. A, 2002, vol. A337, pp. 88-96.

13. O.M. Ivasishin, S.V. Shevchenko, N.L. Vasiliev, and S.L. Semiatin: Acta Mater., 2003, vol. 51, pp. 1019-1034.

14. O.M. Ivasishin, S.V. Shevchenko, P.E. Markovsky, and S.L. Semiatin: Ti-2003: Science and Technology, G. Luetjering and J. Albrecht, eds., WileyVCH Verlag GmbH, 2004, pp. 1307-1314. 
15. O.M. Ivasishin, S.V. Shevchenko, and S.L. Semiatin: Scripta Mater., 2004, vol. 50, pp. 1241-1245.

16. S.L. Semiatin: Unpublished research, Air Force Research Laboratory, Wright-Patterson Air Force Base, OH, 2000

17. A.L. Pilchak, G.A. Sargent, and S.L. Semiatin: Metall. Mater. Trans. A, 2018, vol. 49A, pp. 908-919.

18. F. Bachman, H. Schaeben, and H. Siemes: Solid State Phenom, 2010, vol. 160, pp. 63-68.

19. R.A. Lebensohn and C.N. Tome: Acta metal. mater., 1993, vol. 41(9), pp. 2611-2624.

20. D. Dunst and H. Mecking: Z. Metallk., 1996, vol. 87, pp. 498-507.

21. M. Holscher, D. Raabe, and K. Lucke: Steel Research, 1991, vol. 62(12), pp. 567-575.

22. D. Raabe, F. Roters, and V. Marx: Textures and Microstructures, 1996, vol. 26-27, pp. 611-635.

23. L. Morris, Study of Abnormal Grain Growth in Beta Annealed Ti-6Al-4V Forgings, MS Thesis, Air Force Institute of Technology, 2018, AFITENY-MS-18-M-310. 